\title{
Thierische Säfte und Gewebe in physikalisch-chemischer Beziehung.
}

\author{
V. Mittheilung. \\ Die Resorptions- und Secretionsvorgänge im Allgemeinen. \\ Von \\ Dr. med. Max. Oker-FIom, \\ Willmanstrand (Finnland). \\ (Mit 1 Textfigur.)
}

Wie aus der Ueberschrift ersichtlich, sollen hier die Resorptionsund Secretionsvorgänge im Organismus weder in ihren Einzelheiten erörtert, noch die im Folgenden ausgesprochenen Gedanken mit eigenen speciellen Untersuchungen bezw. Versuchsergebnissen gestützt werden. Ich bezwecke vielmehr mit dieser Mittheilung lediglich, die physikalisch-chemischen Grundlagen der genannten Erscheinungen in groben Zügen zu besprechen, um sodann aus dem schon erbrachten physiologischen Beobachtungsmaterial einige Beispiele von den gewonnenen Gesichtspunkten aus zu beleuchten.

\section{Physikalisch-Chemisches.}

Wenn man über irgend eine Lösung, z. B. aus Kupfersulfat in Wasser, vorsichtig eine Schicht reinen Wassers bringt, so bleibt das Gebilde nicht in Ruhe. Das Kupfersulfat bezw. seine Ionen $\mathrm{Cu}^{+}$ und $\mathrm{SO}_{4}^{-}$beginnen alsbald sich zu erheben, sowie in die Schicht des reinen Wassers zu diffundiren; und ein Gleichgewichtszustand wird erst dann hergestellt, wenn der gelöste Stoff sich gleichmässig in der gesammten Wassermenge vertheilt hat.

Man kann indessen die Diffusion des Kupfersulfates hemmen, wenn man die Lösung vom reinen Lösungsmittel durch eine eingeschaltete Wand trennt, welche den gelösten Stoff nicht durchtreten 
lässt, während sie für das Wasser durchlässig ist. Sobald aber die freie Diffusion des Kupfersulfates durch diese sog. halbdurchlässige oder semipermeable Wand verhindert wird, tritt das reine Wasser nunmehr durch die Wand und mischt sich mit der Kupfersulfatlösung, eine Erscheinung, die Osmose genannt wird, und als deren Ursache der sog. osmotische Druck des gelösten Stoffes angesehen wird.

Von der hypothetischen Natur des osmotischen Druckes werden wir im Folgenden ganz absehen und lediglich an gewissen Erfahrungsthatsachen festhalten.

Der osmotische Druck ist von der Natur des gelösten Stoffes ganz unabhängig; bestimmend ist nur die molekulare Concentration desselben, sowie die vorhandene Temperatur. Für die Elektrolyte spielt hierbei die Dissociation eine grosse Rolle, indem die dissociirten Ionen, jedes für sich, denselben osmotischen Druck wie ein nichtdissociirtes Molekül des Stoffes repräsentiren.

Die Druckwerthe, welche die osmotischen Erscheinungen entfalten können, lassen sich durch directe Manometermessungen feststellen und sind ganz bedeutend. So gibt ${ }^{1}$ ) z. B. eine $1 \%$ ige Zuckerlösung einen Druck von mehr als $500 \mathrm{~mm} \mathrm{Hg}$, sowie eine $1 \%$ ige Zuckerlösung einen solchen von mehr als drei Atmosphären.

Damit aber ein gelöster Stoff den ihm zukommenden Druck entfalten, sowie die entsprechende Arbeit leisten kann, ist es, wie gesagt, eine unerlässliche Bedingung, dass eine Scheidewand, welche für ihn undurehlässig, für das Wasser dagegen durchlässig ist, die Lösung vom reinen Wasser trennt. Solche halbdurchlässige Wände sind in der That dargestellt worden. Bringt man eine Lösung von Kaliumferrocyanid in Berührung mit einer solchen von Kupfersulfat, so scheidet sich an der Berührungsfläche eine membranartige Bildung von Kupferferrocyanid aus, welche unter Anderem das Kupfersulfat nicht durchtreten lässt. Diese an und für sich sehr zarte und zerbrechliche Membran kann auf verschiedene Weise in einem festeren Substrate erzeugt werden, um zu Versuchen geeignete Scheidewände zu erhalten.

Wenn wir nun eine Lösung von Kupfersulfat einer bestimmten Concentration in ein geeignetes, mit der für $\mathrm{CuSO}_{4}$ undurchlässigen Ferrocyankupfermembran versehenes Gefäss geben und die semi-

1) Vgl. W. Ostwald, Grundriss d. allgem. Chemie S. 192.1899. 
permeable Wand andererseits mit einer genügenden Menge reinen Wassers in Berührung bringen, so steigt also die Flüssigkeit im Gefäss bis zu einer gewissen Höhe, deren Betrag dem osmotischen Drucke der Kupfersulfatlösung entspricht. Das Steigen der Kupfersulfatlösung hört erst dann auf, wenn ihm ein entsprechend grosser Gegendruck entgegengesetzt wird. Wird die Lösung aber einem stärkeren Drucke ausgesetzt, so wird durch die semipermeable Membran - die ja für $\mathrm{CuSO}_{4}$ resp. für seine Ionen undurchlässig ist eine Flüssigkeit berausgepresst oder filtrirt, die nur aus reinem Wasser besteht. Hieraus ergibt sich, dass eine Filtrationsbewegung in diesem Sinne, d. h. ein Abtrennen des Lösungsmittels, des Wassers, vom gelösten Stoff einen Druck erfordert, der bedeutender ist als der entsprechende osmotische Druck der Lösung.

Wird eine Wand, die sowohl für den gelösten Stoff wie für das reine Lösungsmittel - wennschon in verschiedenem Grade durchlässig ist, zwischen unsere Flüssigkeiten eingeschaltet, so dringe zunächst beide in entgegengesetzter Richtung durch die Wand hin durch. Passirt das Wasser die Wand leichter, als der gelöste Stofl es zu thun vermag, dann wird sich die Flüssigkeit auf der Seite der! Lösung erst heben, bis der Gehalt der gesammten Flüssigkeitsmenge an ihm, d. h. der osmotische Druck, überall gleich geworden ist. Sobald dies Gleichgewicht der molekularen Concentration eingetreten ist, filtrirt sich die nunmehr gleichmässige Lösung, deren Componenten von der eingeschalteten Wand keine absoluten Bewegungshindernisse erfahren, der herrschenden Druckdifferenz auf beiden Seiten der Wand gehorchend allmälig zurück, bis sie beiderseits gleich hoch zu stehen kommt.

Haben wir es mit gemischten Lösungen zu thun, dann addiren sich die osmotischen Partialdrucke der verschiedenen gelösten Stoffe zu einander. Wird eine solche Lösung vom reinen Lösungsmittel vermittelst einer semipermeablen Membran, welche für sämmtliche gelöste Stoffe undurchlässig ist, getrennt, dann steigt die Lösung zu einer Höhe, welche dem osmotischen Gesammtwerth entspricht. Ist aber die eingeschaltete Scheidewand für gewisse Stoffe der Lösung durchlässig, für andere nicht, so wird die resultirende Steighöhe schliesslich noch von dem osmotischen Partialdruck dieser abhängig sein. Der Einfluss jener wird gewissermaassen auch zur Geltung E. Pfläger, Arehir fūr Physiologie. Ba. 85. 
kommen, indem sie - je nachdem sie schwerer oder leichter die Membran zu durchdringen vermögen - das Eindringen des Wassers in die Lösung erst mehr oder weniger beschleunigen, um in dem Maasse, als sie selber in das reine Wasser austreten und schliesslich zu beiden Seiten der Scheidewand sich in gleicher Concentration vorfinden, das Steigen der Lösung zu verlangsamen. Die Ursache dieser Verlangsamung liegt darin, dass das mit dem - die Scheidewand meistens schwieriger als das reine Wasser passirenden - gelösten Stoff verunreinigte Lösungsmittel durch die Membran langsamer geht als dieses allein.

Betrachten wir wiederum den Filtrationsprocess unter gleichen Umständen, so ergibt sich Folgendes: Wenn die Membran für alle resp. gelösten Stoffe undurchdringlich ist, so kann eine Filtration von nur reinem Wasser hervorgerufen werden; der hierzu nothwendige Filtrationsdruck muss aber den osmotischen Gesammtdruck der Lösung übersteigen. Ist die Membran dagegen für gewisse Stoffe der Lösung durchlässig, für andere nicht, so können jene unter geeignetem Drucke mit dem Wasser filtrirt werden, welche Filtration so lange fortschreitet, bis der osmotische Partialdruck dieser in der Lösung im Laufe der Filtration auf die entsprechende Höhe gestiegen ist. Hieraus erhellt, dass eine Filtration, welche bezweckt, aus einer gemischten Lösung eine Flüssigkeit abzutrennen, welche einen gewissen Bestandtheil der ursprünglichen Lösung nicht enthält, einen Filtrationsdruck erfordert, der höher als der osmotische Partialdruck des betreffenden Bestandtheiles der Lösung ist.

Aus dem oben Angeführten liessen sich noch andere wichtige Schlussfolgerungen berleiten, welche für die Auffassung der Resorptions- und Secretionsvorgänge im lebenden Organismus von besonders grosser Bedeutung wären.

Ich ziehe es jedoch vor, zunächst einige directe Versuche anzuführen.

In der von Paul Walden ${ }^{1}$ ) angegebenen Weise wurde am unteren, plangeschliffenen Ende eines beiderseits offenen, etwa $50 \mathrm{~mm}$

1) Paul Walden, Ueber Diffusionsersckeinungen an Niederschlagsmembranen. Zeitschr. f. phys. Chemie Bd. 10 (6) S. 700-701. 1892. 
langen cylindrischen Glasrohres von etwa $10 \mathrm{~mm}$ innerem Durchmesser durch Eintauchen in eine erwärmte Gelatinelösung $(1: 5)$, welche mit etwas concentrirter Ammoniumchromatlösung versetzt wurde, eine zarte Gelatinemembran erzeugt. Nachdem die Gelatine unter dem Einflusse des Lichtes in die in Wasser schwer lösliche Modification umgewandelt worden war - was durch Bräunung der Membran gekennzeichnet ist -, wurde in ihr sodann eine Ferrocyankupfermembran in der Art erzeugt, dass in das unten geschlossene Röhrchen eine Ferrocyancaliumlösung gebracht und das Röhrchen alsdann für eine Zeit in eine Kupfersulfatlösung getaucht wurde: Nach Abspülen der Membran nebst Röhrchen von anbaftenden Flüssigkeiten war der kleine Apparat zum Gebrauch fertig.

Im Uebrigen war die Versuchsanordnung, die in nebenstebender Figur in natürlicher Grösse abgebildet ist, folgende:

In das unten von der Ferrocyankupfermembran geschlossene Röhrchen $A$ werden etwa $3 \mathrm{~cm}$ einer Kupfersulfatlösung gebracht; die obere Oeffnung des Rohres wird vermittelst eines durchbohrten und mit einer Steigröhre $B$ versehenen Stöpsels luftdicht geschlossen, zu welchem Behufe die Contactflächen zwischen dem Stöpsel und den resp. gläsernen Theilen mit geschmolzenem Wachs übergossen werden.

Beim Einsetzen des Stöpsels wurde die Kupfersulfatlösung etwa 50 bis $70 \mathrm{~mm}$ hoch in die Steigröhre - welche von

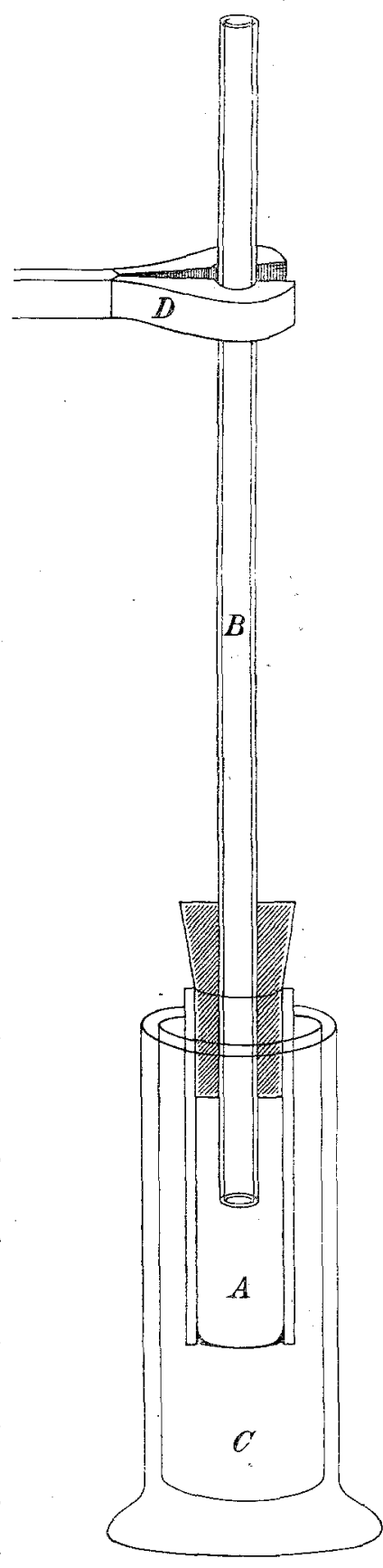

Fig. 1. 36 * 
1 ccm Flüssigkeit auf eine Länge von etwa $123 \mathrm{~mm}$ gefüllt wird getrieben. Das Ganze wird durch eine Klammer $D$ in verticaler Lage gehalten, wobei das Röhrchen $A$ in einem weiteren, etwas $\mathrm{NaCl}$ Lösung enthaltenden Gefässe $O$ zu bängen kommt. Sodann wird das Sinken resp. Steigen des Flüssigkeitsfadens in der Steigröhre während mehrerer Stunden, bezw. Tage beobachtet.

Die Steighöhe des Flüssigkeitsfadens am Anfange des Versuches wird mit 0 bezeichnet; das Steigen bezw. Sinken des Fadens wird mit + bezw. - in mm angegeben.

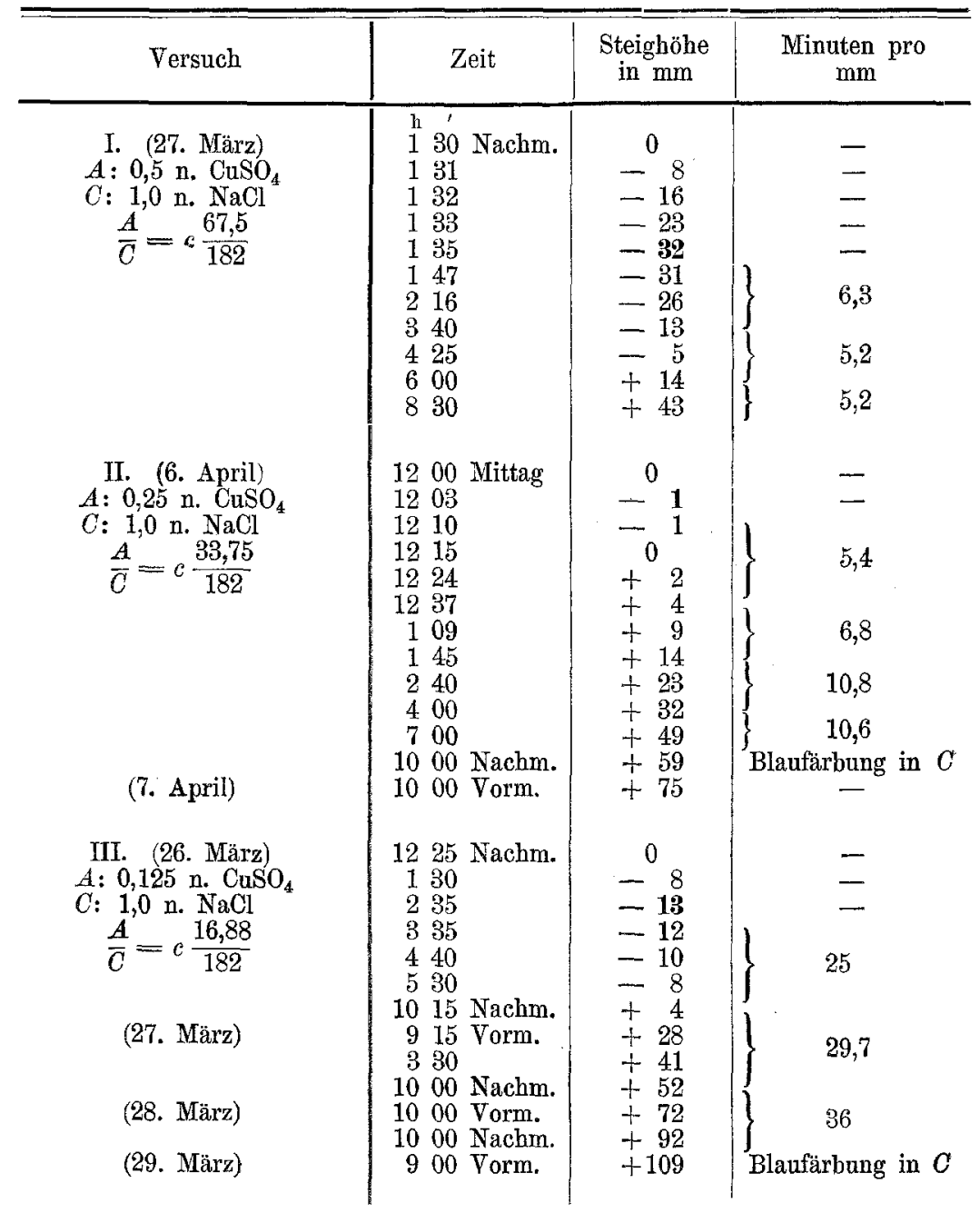


Thierische Säfte und Gewebe in physikalisch-chemischer Beziehung.

\begin{tabular}{|c|c|c|c|}
\hline Versuch & Zeit & $\begin{array}{l}\text { Steighöhe } \\
\text { in } \mathrm{mm}\end{array}$ & $\underset{\mathrm{mm}}{\text { Minuten in }}$ \\
\hline $\begin{array}{c}\text { IV. (10. April) } \\
A: 0,083 \text { n. CuSO } \\
C: \quad 1,0 \text { n. NaCl } \\
\frac{A}{C}=c \frac{11,2}{182} \\
\text { (11. April) } \\
\text { (12. April) } \\
\text { (13. April) } \\
\text { (14. April) } \\
\text { (15. April) } \\
\text { (16. April) }\end{array}$ & $\begin{array}{rll}\text { h } & \text { ' } \\
12 & 51 & \text { Nachm. } \\
1 & 00 & \\
2 & 00 \\
4 & 00 & \\
12 & 00 & \text { Nachm. } \\
9 & 00 & \text { Vorm. } \\
10 & 00 & \text { Nachm. } \\
9 & 00 & \text { Vorm. } \\
9 & 00 & \text { Nachm. } \\
9 & 00 & \text { Vorm. } \\
10 & 00 & \text { Nachm. } \\
11 & 00 & \text { Vorm. } \\
10 & 00 & \text { Nachm. } \\
9 & 00 & \text { Vorm. } \\
9 & 00 & \text { Nachm. } \\
9 & 00 & \text { Vorm. }\end{array}$ & 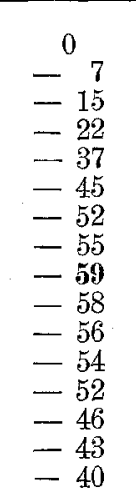 & 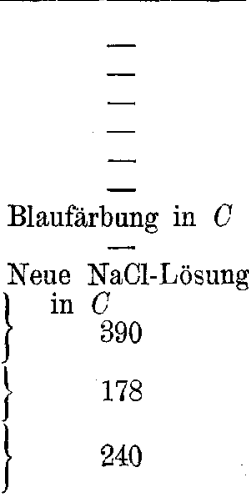 \\
\hline
\end{tabular}

Bei den obigen Versuchen ist die molekulare Concentration der Innenflüssigkeit in $A$ entschieden kleiner als die der Aussenflüssigkeit in $C$. Dementsprechend finden wir bei sämmtlichen Versuchen im Anfange ein Sinken des Flüssigkeitsfadens in $B$, d. h. einen Flüssigkeitsstrom von $A$ nach $C$. Nach einiger Zeit aber hört das Sinken auf und wird sodann allmälig von einem anhaltenden Steigen des Fadens abgelöst. Wie früher hervorgehoben, ist die Ferrocyankupfermembran für $\mathrm{CuSO}_{4}$ resp. seine Ionen $\mathrm{Cu}^{+}$und $\mathrm{SO}_{4}^{-}$undurchlässig, gestattet dagegen dem Wasser wie auch verschiedenen gelösten Salzen, u. A. auch dem $\mathrm{NaCl}$, den Durchtritt. Sofort nach Fertigstellung der Anordnung wird das $\mathrm{NaCl}$ bestrebt sein, in $A$ einzuwandern; da dies aber nicht ganz unbehindert geschehen kann, so wird ein osmotischer Flüssigkeitsstrom von $A$ nach $C$ die Folge sein, da der osmotische Druck in $C$ grösser ist als der in $A$. Inzwischen gelingt es dem $\mathrm{NaCl}$ dennoch, die Membran zu durchdringen, und in dem Maasse, als dies geschieht, wird das Sinken des Flüssigkeitsfadens in $B$ immer langsamer; und sobald die molekulare Concentration der an die Membran grenzenden Schichten beiderseits etwa gleich geworden ist, hört das Sinken ganz auf.

Zum Gleichwerden der molekularen Concentration der resp. Grenzschichten tragen noch folgende Umstände gleichzeitig bei. Durch Entweichen von Wasser aus $A$ durch die Membran wird die entsprechende Wandschicht in $A$ in Bezug auf Kupfersulfat concentrirter, und aus demselben Grunde wird die gegenüberstehende Wandschicht in $C$ in Bezug auf Natriumchlorid weniger concentrirt. 
Sobald nun etwa gleiche molekulare Concentration in den resp. Wandschichten erzielt worden ist, bleibt das Gebilde keineswegs in Ruhe. Das $\mathrm{NaCl}$ trachtet überall, in die gesammte Flüssigkeit sich gleichmässig auszubreiten, und erfährt auch keine unübersteigbare Hindernisse in seinem Bestreben. In Bezug auf $\mathrm{NaCl}$ wird also die Concentration bezw. sein osmotischer Partialdruck früher oder später zu beiden Seiten der Scheidewand gleich.

Anders verhält es sich mit dem Kupfersulfat, für das die Membran undurchlässig ist. Der darauf entfallende osmotische Druck bleibt immerwährend der Flüssigkeit in $A$ vorbehalten und addirt sich zu demjenigen des $\mathrm{NaCl}$ ebendaselbst.

Früher oder später haben wir somit in $A$ einen osmotischen Ueberdruck, der nicht zögert, dureh ein Steigen des Flüssigkeitsfadens in $B$ von seinem Vorhandensein einen sichtbaren Ausdruck zu geben.

Wir finden somit, wie - durch den Einfluss der Scheidewand, welche für $\mathrm{NaCl}$ und Wasser durchlässig ist, für $\mathrm{CuSO}_{4}$ dagegen nicht - der anfänglich gegenüber demjenigen in $C$ niedrigere osmotische Druck in $A$ nach einiger Zeit zunimmt und jenen überragt, sowie die entsprechenden Folgeerscheinungen mit sich bringt. Es kann also eine Lösung, welche einen niedrigeren osmotischen Druck repräsentirt, eine solchevon höherem osmotischem Druck durch osmotische Vorgänge in sich aufnehmen oder resorbiren, sobald die Flüsigkeiten von einer Scheidewand getrennt sind, welche den gelösten Stoff jener nicht durchlässt, während die Bestandtheile dieser die Wand passiren können.

Dies Ergebniss muss von fundamentaler Bedeutung für unsere Auffassung der Resorptions- und Secretionserscheinungen im Allgemeinen sein. Zugleich ergibt sich die ausserordentliche Rolle, welche die membranartigen Gebilde des lebenden Organismus und die osmotisehen Erscheinungen bei den verschiedensten Vorgängen in der organisirten Welt, sowohl im Thier- wie im Pflanzenkörper, spielen.

Ehe wir den zeitlichen Verlauf und etwaige graduelle Verschiedenheiten der Versuche näher betrachten, sei hervorgehoben, dass dieselben einen directen Vergleich nicht unbedingt gestatten; denn die künstlich hergestellten Scheidewände können sowohl in Bezug auf die Dicke der Gelatinehaut wie auf die der Ferrocyan- 
kupfermembran verschieden ausfallen; auch stellt sich mit der Zeit ein störendes Moment dadurch ein, dass die zerbrechliche Membran früher oder später für $\mathrm{CuSO}_{4}$ etwas durchlässig wird, sowie dass sie sich von der Röhrchenwand löst und die Kupfersulfatlösung aus $A$ in $C$ durchsickern lässt. Nur mit der durch diese Versuchsfehler bedingten Reservation wollen also die hier mitzutheilenden näheren Verhältnisse betrachtet sein.

$\mathrm{Da}$ der Dissociationscoëfficient für $\mathrm{NaCl} 0,82$, für $\mathrm{CuSO}_{4}$ dagegen nur 0,35 beträgt, so ist die osmotische Druckdifferenz zwischen den resp. Lösungen bei den Versuchen entschieden grösser, als der Vergleich der resp., in Normalität angegebenen Concentration erscheinen lässt. Das mit Berücksichtigung der Dissociation des resp. Stoffes berechnete Verhältniss findet sich in der ersten Columne der Tabelle angegeben.

Wir entnehmen den Versuchen I-IV, dass das Steigen des erst gesunkenen bezw. eine gewisse Zeit ruhig gestandenen Flüssigkeitsfadens sich desto früher einstellt, je concentrirter die angewandte Kupfersulfatlösung in $A$ ist. Sobald der Faden einmal im Steigen begriffen ist, geschieht dies innerhalb gewisser Grenzen mit annäherungsweise gleichmässiger Geschwindigkeit, welche desto grösser ausfällt, je stärker die Concentration der Kupfersulfatlösung ist.

Wie weit man bei gleichbleibender Concentration der NaClLösung (hier 1,0 normal) mit der Verdünnung der $\mathrm{CuSO}_{4}$-Lösung gehen kann, ohne die resorbirende Wirkung des Kupfersulfates zu vermissen, bleibt - in Anbetracht der besonders langen Zeit, welche die Versuche mit sehr verdünnten Kupfersulfatlösungen in Anspruch nehmen, wobei die Membranen schon gefährdet werden - unentschieden. Eine Kupfersulfatlösung, deren osmotischer Druck, mit demjenigen der Natriumchloridlösung verglichen, durch die resp. Relationen $\frac{67,5}{182}, \frac{33,75}{182}, \frac{16,8}{182}$ und $\frac{11,2}{182}$ angegeben wird, entfaltet noch eine ganz bedeutende resorbirende Wirkung auf diese, und die nach einer gewissen Zeit eintretende Resorption erfolgt mit Geschwindigkeiten, welche mit der Concentration der $\mathrm{CuSO}_{4}$-Lösung Hand in Hand gehen.

Eine 0,5 normale $\mathrm{CuSO}_{4}$ resorbirt noch ganz begierig eine concentrirte NaCl-Lösung, wie aus dem Versuch $\mathrm{V}$ ersichtlich ist. 


\begin{tabular}{|c|c|c|c|}
\hline Versuch & Zeit & $\begin{array}{l}\text { Steighöhe } \\
\text { in mm }\end{array}$ & $\underset{\mathrm{mm}}{\text { Minuten pro }}$ \\
\hline $\begin{array}{c}\text { V. (25. März) } \\
\text { A: } 0, \text { ă n. CuSO } \\
C: \text { conc. NaCl-Lösung } \\
\text { (26. März) }\end{array}$ & 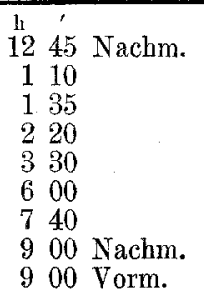 & $\begin{array}{l}0 \\
-\quad 9 \\
-13 \\
-14 \\
-\quad 8 \\
+\quad 4 \\
+\quad 12 \\
+\quad 18 \\
+\quad 70\end{array}$ & $\begin{array}{l}- \\
- \\
11,1 \\
12,5 \\
12,5 \\
13,3 \\
13,8\end{array}$ \\
\hline
\end{tabular}

Dagegen zeigen die obigen Versuche keine Uebereinstimmung zwischen dem Betrag des maximalen Sinkens des Flüssigkeitsfadens und der Concentration der resp. Kupfersulfatlösung, was auf etwaige Ungleichheit der Membranen zurückzuführen ist.

Dass dies thatsächlich der Fall ist, erhellt beim Vergleich der Versuche VI-IX, welche alle vier mit einer und derselben Membran angestellt worden sind.

Wir entnehmen diesen Versuchen nicht nur, dass die Zeit, die bis zum Maximum des Sinkens verstreicht, mit steigender Verdünnung der $\mathrm{CuSO}_{4}$-Lösung zunimmt, sondern auch noch, dass der maximale Werth des Sinkens mit der Verdünnung der Kupfersulfatlösung etwa gleichen Schritt hält.

\begin{tabular}{|c|c|c|}
\hline Versuch & Minuten & $\begin{array}{c}\text { Steighöhe in } \\
\mathrm{mm}\end{array}$ \\
\hline $\begin{array}{c}\mathrm{VI} \\
\mathrm{CuSO}_{4}: 0,5 \mathrm{n} .\end{array}$ & $\begin{array}{r}0 \\
5 \\
10 \\
13 \\
20 \\
36 \\
74\end{array}$ & $\begin{array}{r}0 \\
-\quad 1 \\
+\quad 1 \\
+\quad 2 \\
+\quad 2 \\
+\quad 14 \\
+\quad 14\end{array}$ \\
\hline $\begin{array}{c}\text { VII } \\
\mathrm{CuSO}_{4}: 0,25 \mathrm{n} .\end{array}$ & $\begin{array}{c}0 \\
1 \\
2 \\
7 \\
16 \\
69\end{array}$ & $\begin{array}{c}0 \\
-\quad 1 \\
-\quad 2 \\
-\quad 3 \\
-\quad 2 \\
+\quad 3\end{array}$ \\
\hline $\begin{array}{c}\text { VIII } \\
\mathrm{CuSO}_{4}: 0,125 \mathrm{n} .\end{array}$ & $\begin{array}{r}0 \\
1 \\
2 \\
4 \\
14 \\
\mathbf{9 7} \\
37 \\
57 \\
78\end{array}$ & $\begin{array}{l}0 \\
-\quad 1 \\
-\quad 2 \\
-\quad 4 \\
=\quad 8 \\
=\quad 8 \\
-\quad 8 \\
-\quad 8\end{array}$ \\
\hline
\end{tabular}




\begin{tabular}{c|c|c}
\hline \hline Versuch & Minuten & $\begin{array}{c}\text { Steighöhe in } \\
\text { mm }\end{array}$ \\
\hline \multirow{2}{*}{ IX } & 0 & 0 \\
CuSO $_{4}: 0,0625 \mathrm{n}$. & 1 & -2 \\
& 2 & -3 \\
4 & -5 \\
13 & -10 \\
39 & -20 \\
96 & -44 \\
& 13 St. & -60 \\
& $25 "$ & $-c 70$ \\
& $48 "$ & $-c 70$
\end{tabular}

Dass die Resorptionsgeschwindigkeit nicht nur von der Concentration der Kupfersulfatlösung, sondern auch von derjenigen der zu resorbirenden Lösung beeinflusst wird, ergibt sich aus der einfachen Thatsache, dass diese mit steigender Concentration von der Membran immer grössere Bewegungshindernisse erfährt, wie es auch die Versuche XI und XII illustriren mögen.

\begin{tabular}{|c|c|c|c|}
\hline Versuch & Minuten & $\begin{array}{l}\text { Steighöhe } \\
\text { in mm }\end{array}$ & $\underset{\text { Minuten pro }}{\mathrm{mm}}$ \\
\hline $\begin{array}{l}\text { X } \\
\text { A: } 0,083 \text { n. } \mathrm{CuSO}_{4} \\
C: \text { Aqua destill. }\end{array}$ & $\begin{array}{r}0 \\
30 \\
90 \\
150 \\
176 \\
221\end{array}$ & $\begin{array}{r}0 \\
+\quad 1 \\
+\quad 6 \\
+\quad 11 \\
+13 \\
+15\end{array}$ & $\begin{array}{l}13,6 \\
21,9\end{array}$ \\
\hline $\begin{array}{c}\mathrm{XI} \\
A:\left\{\begin{array}{c}0,083 \mathrm{n} \cdot \mathrm{CuSO}_{4} \\
0,89 \mathrm{n} \cdot \mathrm{NaCl}\end{array}\right. \\
C: 0,83 \text { n. } \mathrm{NaCl}\end{array}$ & $\begin{array}{r}0 \\
39 \\
72 \\
312 \\
987\end{array}$ & $\begin{array}{l}0 \\
+\quad 1 \\
+\quad 3 \\
+\quad 12 \\
+\quad 38\end{array}$ & $\begin{array}{l}26 \\
27,1\end{array}$ \\
\hline
\end{tabular}

Auf Grund obiger Versuchsergebnisse und Betrachtungen finden wir somit, dass, wenn zwei Flüssigkeiten durch eine Scheidewand von einander getrennt sind, welche für alle Bestandtheile der einen Flüssigkeit durchlässig, dagegen für einen gewissen gelösten Stoff der anderen undurchlässig ist, der osmotische Druck eben dieses Stoffes schliesslich das Geschick des Gebildes lenken wird.

Um Verwechselungen zu vermeiden, dürfte es sich empfehlen, die schliesslich wirksame osmotische Druckcomponente des Gebildes als Resorptionsdruck zu bezeichnen, unter welchem Ausdruck wir also einen osmotischen Druck verstehen würden, welcher - den 
Partialdruck eines oder mehrerer gelöster Stoffe darstellend - vom resp. osmotischen Partialdrucke der übrigen Bestandtheile, sowie ganz besonders von den verschieden grossen Bewegungshindernissen, die eine eingeschaltete halbdurchlässige Scheidewand den sämmtlichen Bestandtheilen der betreffenden Flüssigkeiten entgegensetzt, abhängig ist.

\section{Die Resorption aus den serösen Höhlen.}

Bei der in der letzten Zeit näher untersuchten Resorption von Flüssigkeiten resp. Lösungen aus den serösen Höhlen sind manche in physikalisch-chemischer Beziehung interessante Ergebnisse erzielt worden. So hat Hamburger ${ }^{1}$ ) gefunden, dass eine in die Bauchhöhle eingeführte, mit dem Blutplasma des Versuchsthieres isotonische Lösung ihre molekulare Concentration bis zur stattgefundenen Resorption nicht ändert. Eine gegenüber dem Blutplasma hyperisotonische Lösung wird während des Resorptionsprocesses in Bezug auf ihre Concentration geschwächt, bis diese der molekularen Concentration des Blutplasmas etwa gleichkommt, wonach die Resorption, wie im vorigen Falle, ohne weitere Aenderungen der Concentration der intraperitonealen Lösung sich vollzieht. Vice versa nimmt eine hypotonische, in die Bauchhöhle eingeführte Lösung im Laufe der Resorption meistens die dem Blutplasma entsprechende Concentration an.

Die Erklärung dieser Concentrationsänderungen der intraperitonealen Lösungen, welche ein Concentrationsgleichgewicht dieser mit dem Blutplasma herzustellen streben, ist auch bei den Physiologen keinen Schwierigkeiten begegnet, und zwar sind die genannten Vorgänge auf einen osmotischen Austausch zwischen den Bestandtheilen der fraglichen Flüssigkeiten, d. h. sowohl der gelösten Stoffe wie des Lösungsmittels der injicirten Lösung und derjenigen des Blutes, zurückgeführt worden. Haben ja auch directe Analysen ergeben, dass nach Einführung in die Bauchhöhle von z. B. einer mit dem Blutplasma isotonischen $\mathrm{Na}_{2} \mathrm{SO}_{4}$-Lösung die isotonisch bleibende intraperitoneale Lösung nach einiger Zeit erhebliche Mengen $\mathrm{NaCl}$ und in geringerem Grade auch andere gelöste Bestandtheile des Blutes enthält.

Nach den meisten Autoren wird die Hauptrolle bei der Resorp-

1) Pflüger's Archiv Bd. 56 S. 572. 1894. 
tion von Lösungen aus den serösen Höhlen den Blutgefässen zugeschrieben, während die Lymphgefässe bei der Resorption von festen Körperchen (Milchkugeln u. s. w.) sich betheiligen sollen. Wir setzen also voraus, dass die Behauptung, die Lösungen seien aus der Bauchhöhle von den Blutgefässen resorbirt, richtig ist. Wie kommt es aber dann vor, dass eine mit dem entsprechenden Blutplasma isotonische Lösung von den Blutgefässen oder richtiger vom Blute aufgenommen wird? Die Antwort lautet: Dies ist schlechthin ein Erforderniss der bestehenden physikalisch-chemischen Verhältnisse.

Es hat sich herausgestellt, dass die in Betracht kommenden Blutgefässwände für die gelösten Stoffe der injicirten Flüssigkeit wie auch für die Mehrzahl der gelösten Bestandtheile des Blutes und für das Lösungsmittel, das Wasser, durchlässig sind. Sobald nun alle diese durch die Gefässwände sich ausgeglichen haben, ist wohl die molekulare Concentration ausser- wie innerhalb der Gefässe etwa dieselbe, $d . h$. der osmotische Druck in der intraperitonealen Flüssigkeit und dem Blutplasma ist gleich, insofern er sich nur auf die resp. Flüssigkeiten als solche bezieht. Dies genügt uns aber bei Weitem nicht.

Der osmotische Druck kommt überhaupt zum Ausdruck, d. h. er kann nur unter der Bedingung in nützliche Arbeit umgesetzt werden, dass eine Wand, welche für den gelösten Stoff undurchdringlich ist, zwischen der fraglichen Lösung und einer schwächeren solchen bezw. dem reinen Lösungsmittel eingeschaltet ist. Sobald in unserem Falle die molekulare Concentration der durch die Gefässwand von einander getrennten Flüssigkeiten durch gegenseitigen Austausch hergestellt wird, haben wir im Blute dennoch Stoffe, welche in der intraperitonealen Flüssigkeit (im selben Unfange jedenfalls) vermisst werden, und zwar betrifft dies ganz besonders die Eiweissstoffe des Blutplasmas, welche die Gefässwand unter physiologischen Verhältnissen nicht oder beinahe nicht durchdringen können. Diese sind es nun, welche schliesslich eine osmotische Arbeit vollziehen können oder richtiger müssen, ganz wie es das Kupfersulfat der relativ verdünnten Lösung gegenüber der vielmal concentrirteren Natriumchloridlösung thut, welche durch eine Ferrocyankupfermembran, die für das $\mathrm{CuSO}_{4}$ nicht oder schwer passirbar, für das $\mathrm{NaCl}$ dagegen leicht passirbar ist, von einander getrennt sind.

Den osmotischen Partialdruck der gelösten Eiweissstoffe im 
Plasma von Pferdeblut hat $G$. Tamman $n^{x}$ ) bei Bluttemperatur auf höchstens $6 \mathrm{~mm}$ Quecksilber berechnet, was einem Gehalt des Plasmas an Eiweissstoffen von etwa $7 \%$ entspricht. Wohl ist dieser Betrag nicht gross, wenn er mit dem Partialdruck der gesammten anorganischen Stoffe des Pferdeblutserums, welcher auf etwa $5000 \mathrm{~mm}$ ( $=6,6$ Atmosphären) angeschlagen worden, verglichen wird. Indessen dürfte vorläufig noch nicht festgestellt sein, inwieweit die betreffenden Gefässwände im normalen Zustande für alle anderen gelösten organischen Bestandtheile des Blutes passirbar sind oder nicht. Den auf die gesammte Menge der organischen Stoffe des Pferdeblutplasmas bezogenen osmotischen Partialdruck hat Tammann zu $840 \mathrm{~mm}$ (= 1,1 Atmosphären) berechnet.

Wie es sich mit der Grösse des osmotischen Druckes derjenigen gelösten Bestandtheile des Blutes verhalten mag, welche von der physiologischen Gefässwand zurückgehalten werden, kann vorläufig dahingestellt bleiben. Unter allen Umständen ist der osmotische Ueberdruck vorhanden und muss seine Wirkung entfalten.

Es ist von vorne herein nicht ganz sicher, dass die molekulare Concentration der injicirten Flüssigkeit im Laufe der Resorption derjenigen des Blutplasmas bezw. des Blutes absolut gleich wird. Die Methoden, die wir besitzen, um dies zu ermitteln, d. h. hier lediglich die Bestimmung der Gefrierpunktserniedrigungen, sind kaum empfindlich genug, um einen kleinen Ueberdruck, welcher den Eiweissstoffen des Blutes entsprechen würde, mit Sicherheit hervortreten zu lassen. Dies ist aber auch nicht gerade nöthig. Wir haben beim Versuche mit der Ferrocyankupfermembran gesehen, dass auch eine Lösung von höherem osmotischem Gesammtdruck durch eine semipermeable Membran von einer solchen von niedrigerem osmotischem Druck aufgenommen wird, sobald diese einen Stoff in Lösung enthält, welcher die Membran nicht zu durchdringen vermag, wäbrend die gelösten Stoffe jener dies thun können. Bei welcher Stufe der gegenseitigen Concentrationsverhältnisse die durch eine halbdurchlässige Wand von einander getrennten Lösungsschichten ein gleichmässiges Aufnehmen der einen Lösung in die andere beginnt, dürfte zuletzt von den relativen Hindernissen abhängen, die dem Lösungsmittel und den gelösten Stoffen, welche durchzudringen vermögen, von der

1) G. Tamman, Die Thätigkeit der Niere im Lichte der Theorie des osmotischen Druckes. Zeitschr. f. physik. Chemie Bd. 20 (2) S. 184 f. 1896. 
Scheidewand entgegengesetzt werden. Hierzu brauchen aber nur die Grenzschichten zu beiden Seiten der Scheidewand, nicht aber die ganze Menge der in mittelbare Berührung mit einander gebrachten Flüssigkeiten die erforderlichen Concentrationsverhältnisse zu erfüllen. Wir können somit nicht umhin, in den im circulirenden Blute gelösten Eiweiss- und eventuell auch anderen Stoffen sowie in der Figenschaft der betreffenden Gefässwände, in normalem Zustande für.dieselbe undurchlässig zu sein, die physikalische Ursache zu erblicken, auf Grund welcher die Resorption einer mit dem entsprechenden Blutplasma sowohl hypo-, isowie hypertonischen Wasserlösung von Stoffen, welche die Gefässwand bezw. das Peritoneum zu durchdringen vermögen, aus der Bauchhöhle schliesslich stattfinden $\mathrm{muss}$.

Allerdings kann gegen die Behauptung, dass die Gefässwände für die im Blutplasma enthaltenen Eiweissstoffe undurchlässig wären, der Einwand erhoben werden, dass auch Eiweissstoffe in eine injicirte intraperitoneale, z. B. $\mathrm{NaSO}_{4}$-Lösung austreten. Es fragt sich aber, in welchem Grade dies stattfindet, sowie ob sie vom Blutplasma herrühren, und $o b$ sie eine völlig unversehrte Gefässwand durchdrungen haben.

Es scheint mir, dass wir einen gewissen Anhaltspunkt für das thatsächliche Verhalten im Folgenden haben. Nach chemischen Analysen von Schmidt ${ }^{1}$, Hoppe-Seyler und Scherer fällt die Menge der festen Bestandtheile der pleuritischen Transsudate sehr verschieden $(36,05$ bis 64,48 auf 1000$)$ aus, was vom verschiedenen Gehalt an Eiweissstoffen herrührt, während die Concentration des Transsudates an anorganischen Salzen lediglich in engen Grenzen (7,55-7,98 auf 1000) schwankt und derjenigen des Blutplasmas etwa gleichkommt. Sehen wir von dem entzündlichen oder exsudativen Processe der Pleurahöhle, sowie von krankhaften Veränderungen der betreffenden Blutgefässe ab, so wird das pleuritische Transsudat (Hydrotorax) am häufigsten durch Stauungen im Kreislaufe in Folge von Herz- resp. Respirationskrankheiten veranlasst. Sobald der Abfluss des Blutes aus der Vena cava superior

1) H. Eichhorst, Handb. d. speciellen Pathologie und Therapie Bd. 1 S. 600.1887 . 
nach dem Herzen stockt und dementsprechend das Blut in den Venae azygos und hemiazygos unter abnorm hohen Druck zu stehen kommt, wird ein transsudativer Ausguss in der Pleurahöhle die Folge sein. Wir können uns leicht denken, dass der Druck, unter welchen das Blut in den grossen Brustvenen zu stehen kommt, wechselnde Höhe erreichen kann; und dennoch finden wir die Concentration des Transsudates in Bezug auf die anorganischen Salze ziemlich constant. Dies würde dafür sprechen, dass ein relativ unbedeutend gesteigerter Blutdruck ausreichen würde, um das Salzserum des Blutes durch die Gefässwände auszutreiben, und es würde sich dementsprechend das Wasser des Serums nebst gelösten anorganischen Salzen relativ leicht durch die Gefässwände filtriren können. Dagegen dürfte der wechselnde Gehalt des Transsudates an Eiweissstoffen in nahen Zusanmenhang mit den im venösen System herrschenden grösseren Druckveränderungen gebracht werden, welches wiederum sich dahin deuten lässt, dass das Eiweiss erst bei höher gesteigertem Drucke austritt und somit die Gefässwand nur schwer, schliesslich aber doch durchdringen kann. Dass die Gefässwände an verschiedenen Körperstellen für die gelösten Bestandtheile des Blutes in verschiedenem Grade durchlässig sind, scheint daraus hervorzugehen, dass, wenn an einer Person zugleich an mehreren Orten Transsudate vorkommen, dasjenige der Pleurahöhle am meisten feste Bestandtheile enthält, und dass dann nach einander das der Peritonealböhle, das der Hirnventrikeln und dasjenige des subcutanen Zellgewebes folgen.

Ungeachtet der sehr unbedeutenden Höhe der den Eiweissstoffen des Blutplasmas entsprechenden osmotischen Druckes darf uns die grosse Resorptionsfähigkeit der serösen Räume nicht befremden; denn mit dem Blutstrome werden die resorbirten Flüssigkeitsmengen immerfort weggeschafft und auf die ganze Blutmasse vertheilt, resp. aus dem Körper ausgeschieden, während die inneren Gefässwände unauf hörlich von frischen Mengen gelöster Eiweisskörper gespült werden. Haben ja auch Versuche von Hamburger dargethan, dass die Durchspülung der Blutgefässe eines todten Thieres mit frischem Serum die Resorption sehr wesentlich befördert.

$\mathrm{Ob}$ zu dem oben skizzirten, die serösen Höhlen betreffenden Resorptionsmechanismus noch eine besondere Zellenthätigkeit ausser derjenigen, die in der Eigenschaft der betreffenden Gewebe besteht, einige Stoffe durchtreten zu lassen, anderen aber unüber- 
Thierische Säte und Gewebe in physikalisch-chemischer Beziehung. 559

steigbare Hindernisse in den Weg zu setzen - hinzutritt, erscheint vorläufig sehr fraglich.

\section{Die Resorption aus dem Darme.}

Bei der folgenden Betrachtung lassen wir die Resorption der Fette ganz bei Seite und beschäftigen uns nur mit der der Eiweissstoffe, Salze und Kohlehydrate resp. ihrer Lösungen.

Was von Ham burger bezüglich der molekularen Concentrationsänderungen der zu resorbirenden Lösungen für die serösen Räume nachgewiesen ist, hat Höber auch bezüglich der Resorption im Dünndarm als gültig gefunden. Es stellte sich nämlich heraus, dass Lösungen verschiedener Salze, die gegenüber dem betreffenden Blutplasma entweder hyper- oder hypotonisch waren, im Laufe der Resorption eine mit thm isotonische Contraction annahmen. Ferner fand $\mathrm{Höber}$, dass unter einander isotonische Lösungen verschiedener Salze aus dem Darme verschieden rasch resorbirt wurden, welcher Umstand für eine verschiedene Durchlässigkeit der in Frage kommenden Gewebeelemente für die verschiedenen Stoffe resp. ihre dissociirten Ionen spricht.

Wir gehen bei unserer Betrachtung von der allgemein anerkannten Behauptung aus, dass die Resorption der angegebenen Stoffe in der einen oder anderen Weise in die Blutgefässe (beinahe ausschliesslich) und zwar in das Wurzelgebiet der Pfortader geschieht. Wir sehen zunächst von einer speciellen Thätigkeit der zelligen Elemente der Schleimhaut des Verdauungsrohres ab und wollen lediglich die physikalischen Bedingungen der Resorption aus dem Darme näher in's Auge fassen.

Nach dem angeführten Ergebnisse Höber's scheinen sie in Bezug auf Salzlösungen denjenigen bei der Resorption aus den serösen Höhlen recht analog zu sein. Wir müssen daher annehmen, dass die Gewebeelemente, welche die gelösten Stoffe der zu resorbirenden Flüssigkeit vom Blutplasma trennen, für jene mehr oder weniger durchlässig sind. Dann werden wir nach hergestelltem Gleichgewicht zwischen den Stoffen, welche die fraglichen Gewebe zu durchdringen vermögen, und dem Lösungsmittel der Resorptionsflüssigkeit und des Blutplasmas wiederum einen den gelösten Eiweisskörpern des

1) Pfläger's Archiv Bd. 70 S. 624. 1898. 
Blutes entsprechenden osmotischen Ueberdruck auf der Seite des Blutes haben. Da nun diese die trennende Zwischenwand nicht durchdringen dürften, müssen sie eine osmotische Arbeit leisten, welche auf die allmälige Aufsaugung der im Darme befindlichen Lösung abzielt.

Was diejenigen Eiweisskörper betrifft, die dem Körper durch die Nahrung zugeführt werden, so werden sie ja bekanntlich unter Einwirkung der Verdauungssäfte hydrolytisch gespaltet. Diese Spaltungsproducte, Peptone und Albumose, werden von den im Verdauungsrohre befindlichen (und daselbst ausgegossenen) Flüssigkeiten in Lösung gebracht.

Während die nativen Eiweissstoffe wenig oder gar nicht diffusible Körper darstellen, zeichnen sich die Albumosen und die Peptone dadurch aus, dass sie - besonders die Peptone - durch vegetabilisches Pergament diffundiren können, was mit ihren gegenüber den Muttersubstanzen relativ kleinen Molekülen in Zusammenhang $\mathrm{zu}$ bringen wäre.

Auf die eine oder andere Weise werden nun die verdauten Eiweissstoffe aus der Darmböhle vom Blute aufgenommen. $\mathrm{Zu}$ bemerken ist aber, dass kein Pepton im Blute zu finden ist; nicht einmal nach der Verdauung von grösseren Fiweissmengen hat man im Pfortaderblute Pepton finden können. Der osmotische Partialdruck des Peptons im Blute ist somit gleich Null, woher das Eindiffundiren der Peptone durch die betreffenden Scheidewände immerwährend unter dem vollen, ihrem Gehalte in der Darmflüssigkeit entsprechenden osmotischen Drucke verlaufen kann, was für ihre Resorption von besonderer Bedeutung sein muss.

Wo oder in welchen Zellen diese Umwandlung des Peptons geschieht, mag dahingestellt bleiben. Unter allen Umständen muss sie vom physikalisch-chemischen Gesichtspunkte aus beim Resorptionsvorgange eine durchgreifende Rolle spielen.

Auf die Bedeutung der Umwandlung für die Resorption der Eiweissstoffe selber ist eben hingewiesen worden. Eine andere, nicht zu unterschätzende Bedeutung scheint mir im Folgenden zu liegen. Sobald die diffusiblen Peptone die Darmhöhle verlassen, werden sie sofort zu complicirteren Eiweissverbindungen synthetisirt; hierbei büssen sie aber sogleich ihre Eigenschaft, durch die Wand zurückpassiren zu können, ein. Nach dieser Umwandlung tragen sie - in die Blutbahn resp. in die Zellenelemente einmal gelangt - zum 
Tbierische Säfte und Gewebe in physikalisch-chemischer Beziehung. 561

Resorptionsdrucke der gelösten Eiweissstoffe des Blutes bei. Obgleich sie immerwährend vom Blutstrome fortbefördert werden, bilden sie bei einer gleichmässigen Resorption in den Gefässen jedenfalls eine etwas concentrirtere Wandschicht von Eiweissstoffen und können somit die nützliche Arbeit dieser in Bezug auf die Resorption von anderen gelösten Stoffen, für welche die Darmwand durchlässig ist, gewissermaassen unterstützen.

$O b$ wir für das Verständniss der Eiweiss- und Salzresorption vom Darme aus eine besondere Zellenthätigkeit zu Hülfe zu nehmen brauchen, scheint auf Grund der obigen Betrachtung vom physikalischchemischen Standpunkte aus verneint werden zu müssen.

Wenn eine specielle Resorptionsthätigkeit der Zellen der Darmschleimhaut vorhanden ist, so muss sie die Verdauungsproducte erst selber aufnehmen, d. h. ihre an das Darmlumen grenzende Wand muss für die genannten Stoffe durchlässig, sowie ihre gegen die tieferen Gewebe resp. gegen die Blutgefässe gerichtete Wand für die von ihnen eventuell bewerkstelligten Umwandiungsproducte durchlässig sein. Wenn nun einige von den Umwandlungsproducten die gegen das Darmlumen gerichtete Zellenwand nicht passiren können, so haben wir wiederum die physikalischen Bedingungen, welche eine osmotische Arbeit im Sinne der Resorption herbeiführen müssen. Hierdurch wäre der Mechanismus der Resorption nicht viel geändert; nur wäre an Stelle der Gefässwand die Bedeutung der gegen das Darmlumen gerichteten Wand der Schleimhautzelle in den Vordergrund geschoben. Die specielle Zellenthätigkeit bei der Resorption würde demnach darin bestehen, aus dem vom Darme aufgenommenen Material Stoffe zu erzeugen, für welche der Rücktritt in die Darmhöhle nicht weiter möglich ist. Ohne allen Zweifel sind alle Zellen des Organismus für Wasser durchlässig, - wenn auch einzelne nur in einer Richtung. Ebenso sichergestellt dürfte es sein, dass die organisirten Gewebeelemente für verschiedene gelöste Stoffe in sehr verschiedenem Grade durchlässig sind, obgleich unsere Kenntnisse auf diesem Gebiete heutzutage noch sehr dürftig sind. Da nun im Blute Stoffe vorhanden sind, welche als besonders wenig diffusible gelten, während die Bestandtheile des Darminhaltes solche gelöste Stoffe nicht oder jedenfalls in geringerem Grade beherbergen, und hierbei die zwischenliegende Gewebeschicht für das Lösungsmittel, das Wasser, durchlässig ist, kann man nicht umhin, einige 
gegenseitige osmotische Beziehungen zwischen dem Inhalte des Darmrohres und der betreffenden Blutgefässe anzunehmen.

Bekanntlich ist die Resorptionsfähigkeit in verschiedenen $\mathrm{Ab}$ theilungen des Verdauungsrohres eine sehr verschiedene. Bei einem Druck von $10 \mathrm{~cm}$ Wasser soll beim Hunde von einem Centimeter der Darmlänge pro Stunde vom Dickdarm etwa 2,1, vom unteren Theil des Dünndarmes etwa 1,3 und vom oberen Theil etwa $0,7 \mathrm{ccm}$ von einer $0,6 \%$ igen Kochsalzlösung resorbirt werden. Wenn in der Kochsalzlösung auch etwas Eiweissstoffe zugegen wären, könnte die verschiedene Resorptionsfähigkeit der verschiedenen Darmabtheilungen sehr wohl damit zusammenhängen, dass diese Stoffe im oberen Theil des Darmrohres zur grossen Menge noch ungespalten sind, und dass sie mit dem Fortrücken längs des Darmeanals von den Verdauungssäften immer mehr hydrolisirt und in die diffusiblen Albumosen und Peptone übergeführt worden. Gewiss könnten ja auch andere wechselnde Bedingungen, wie verschiedene Durchlässigkeit u. s. w., in Betracht gezogen werden.

Wir finden-somit, dass bei der Resorption vom Verdaungsrohre die physikalischen Bedingungen für eine rein osmotische Aufsaugung vorhanden sind. Eine ganz besondere Rolle scheint hierbei den verdauten Eiweissproducten zuzukommen, indem sie sogleich nach dem Verlassen des Darmlumens ihren diffusiblen Charakter einbüssen und somit zur Erhöhung des Resorptionsdruckes der gelösten Eiweisskörper des Blutes beitragen. Wo wir die für den osmotischen Resorptionsvorgang so bedeutungsvolle Scheidewand zu suchen haben, in den Schleimhautzellen oder in der Gefässwand, wird nicht leicht zu entscheiden sein. Einen wichtigen Anhaltspunkt in dieser Beziebung würde der sichere Nachweis der Umwandlungsstätte der Peptone in die Bluteiweisskörper darbieten. Bei directer Einspritzung von Pepton in die Blutbahn scheint dasselbe schnell aus dem Blute zu verschwinden; durch Digestion von Blut mit Pepton in vitro soll dies nicht eintreten, was dafür zu sprechen scheint, dass die Umwandlung der Peptone in die Bluteiweissstoffe nicht im Blute selber stattfindet.

Die Art der Resorption der Kohlehydrate resp. des Zuckers aus dem Darme dürte nicht fern von der der Salze sein. Da das normale Blut sehr wenig Zucker enthält und der resorbirte Zucker immerwährend mit dem Pfortaderblute weiter transportirt wird, kann 
es die ganze Zeit, seinem - beinahe unverkürzten - eigenen osmotischen Druck im Darminhalte entsprechend, in die Blutbahn streben.

Was schliesslich die Verdauungsenzyme selber betrifft - die ja auch Eiweisskörper darstellen -, so gehen sie als solche in den Kreislauf über und werden ebenso unverändert mit dem Harn ausgeschieden. Sie stellen also durch die betreffenden Gewebeelemente diffusible Stoffe dar und können folglich keine Gegenwirkung gegen die osmotische Arbeit der gelösten Bluteiweissstoffe entfalten.

Auch bezüglich der Gallenbestandtheile, die ebenfalls leicht resorbirt werden, dürfte das eben Gesagte gelten.

\section{Die Secretionsthätigkeit der Speicheldrüsen.}

Mit der Thatsache vor Augen, dass die Secretionsproducte der Speicheldrüsen Stoffe enthalten, die weder im Blute noch in der Lymphe zu finden sind, dürfte wohl Niemand den betreffenden Drüsenzellen eine besondere specifische Thätigkeit bei der Secretion absprechen wollen. Haben ja auch Heidenhain, Langley, Biedermann u. A. gezeigt, dass sowohl die Eiweissdrüsen als auch die Schleimdrüsen bei stattfindender Secretion morphologischen Veränderungen unterliegen, welche in dem Sinne gedeutet werden müssen, dass in den Drüsenzellen specifische Substanzen, complicirte Eiweissverbindungen gebildet und während der Secretion sodann ausgeschieden werden.

Ebenso dürfte wohl auch Niemand behaupten wollen, dass die anorganischen Salze, die wir im Speichel antreffen, in den Drüsenzellen im Allgemeinen besondere chemische Umwandlungen erfahren würden, oder dass selbst das Wasser von ihnen neu gebildet worden wäre; vielmebr dürfte dem thatsächlichen Verhalten wohl die Vermuthung entsprechen, dass sie von den Drüsen als solches Material selber irgendwie aufgenommen wären. Wir müssen somit annehmen, dass die meisten Salze, sowie das Wasser des secernirten Speichels vom Nährmaterial der Drüsen herrühren, und dass folglich Bedingungen vorhanden sein müssen, unter denen gewisse Gewebeelemente der betreffenden Drüse für die genannten Stoffe durchlässig sind. Wenn dies einmal der Fall ist, können wir nicht umhin, osmotische Beziehungen zwischen den Secretionsproducten und der Nährflüssigkeit zur Geltung kommen zu lassen.

Um die osmotischen Vorgänge hierbei überblicken zu können, 
wäre zunächst $\mathrm{zu}$ entscheiden, woher die Drüsen das Material zur Bereitung der Secretionsproducte beziehen. Der Umstand, dass die Speichelsecretion ziemlich unabhängig von dem in den Drüsencapillaren herrschenden Blutdrucke. stattfindet, spricht eigentlich weder für noch gegen die Annahme, dass das Blut das Material liefern würde; denn zur Bildung ihrer specifischen Secretionsproducte dürften die Drüsenzellen wohl unter den verschiedenen Druckverhältnissen dennoch Nahrung genug erhalten, und sobald diese Stoffe einmal da sind, erleiden die osmotischen Beziehungen zwischen den resp. Flüssigkeiten von den Blutdruckvariationen keine besonderen Finflüsse. Dagegen spricht die Thatsache, dass bei verbluteten Thieren - selbst wenn durchschnittene grössere Arterien keinen Tropfen Blut mehr geben - die Reizung der Absonderungsnerven eine Speichelsecretion hervorruft, dafür, dass die Speicheldrüsen ihr Rohmaterial nicht vom Blute beziehen. Durch dies Verhalten dürfte wohl auch, wie Tigerstedt ${ }^{1}$ ) hervorhebt, jeder Verdacht, die Speichelabsonderung als einen einfachen Filtrationsvorgang vom Blute aus aufzufassen, ein für alle Mal beseitigt sein. Gegen eine solche Auffassung spricht bierbei noch ganz entschieden der Umstand, dass der Manometerdruck im Ausführungsgange der Submaxillarisdrüse bei Reizung der cerebralen Absonderungsnerven in sehr kurzer Zeit bis zu etwa $100 \mathrm{~mm} \mathrm{Hg}$ höher als der in der A. carotis vorhandene getrieben werden kann. In Anbetracht dessen, dass unter dem im Abführungsorgan obwaltenden hohen Drucke eine Filtration von innen nach aussen durch die Drüsengänge statthaben kann, entspricht, wie Tigerstedt bemerkt, der so gemessene Druck noch nicht dem Maximum der von den Drüsenzellen entwickelten Druckkräfte.

So entschieden der bohe Absonderungsdruck gegen eine einfache Filtration vom Blute aus spricht, so wenig darf die Höhe des Druckes befremden, wenn wir bedenken, dass wir es mit osmotischen Erscheinungen zu thun haben können. Wissen wir doch, dass osmotische Erscheinungen ganz ausserordentlich hohe, bis zu mehreren Atmosphären steigende Druckwerthe erzeugen können.

Um im Drüsenausführungsgang einen osmotischen Druck in der angegebenen Richtung bekommen zu können, müssen wir 1. auf der Seite des Drüsenlumens lösliche Stoffe haben, welche in der Nährflüssigkeit nicht oder in geringerer Concentration vorkommen, und

1) Tigerstedt, Lehrb. d. Physiologie des Menschen Bd. 1 s. 287. 1897. 
2. muss eine Scheidewand, welche für diese Stoffe nicht oder wenig durchlässig ist, sie von der Nährflüssigkeit trennen.

Was nun diese betrifft, so dürfte es nicht fern liegen, an die specifischen Absonderungsproducte der Drüse zu denken, wobei sich mir sogleich eines aufdrängt, welches in Bezug auf die Thätigkeit der Schleimdrüsen eventuell von Bedeutung sein könnte. Ich meine das Mucin, welches zu den zusammengesetzten Eiweisskörpern, den Proteiden, gehört und somit grōssere Moleküle besitzt als die einfachen Eiweisskörper. Die für die osmotischen Drucke so bedeutungsvolle halb durchlässige Membran muss selbstverständlich irgendwo zwischen der Nährflüssigkeit und der Stätte des Entstehens der specifischen Absonderungsproducte liegen. Da nun diese Producte mit aller Wahrscheinlichkeit in den Drüsenzellen entstehen, bleibt nichts Anderes übrig, als die halb durchlässige Membran in die gegen die Nährflüssigkeit bezw. gegen die Lymphräume gerichtete Zellenwand, resp. in die Membrana propria zu verlegen.

Ob die specifischen Secretbestandtheile der Speicheldrüsen bezw. der Schleimdrüsen Abkömmlinge des lebendigen Protoplasmas oder Producte von dessen Thätigkeit darstellen, mag als bisher nicht entscheidbar dahingestellt bleiben; irgendwo müssen die Zellen das zum Aufbau der abzusondernden complicirten Eiweisskörper nöthige Material beziehen. Ob nun dies Material von den Eiweissstoffen der Nährflüssigkeit als solche geliefert oder als Spaltungsproducte derselben von den betreffenden Zellen aufgenommen wird: die erste Bedingung wird jedenfalls die sein, dass diese Stoffe in innige Berührung mit dem Zellprotoplasma kommen, d. b. ihre gegen die Nährffüssigkeit gerichtete Wand nebst Membrana propria durchdringen können. Wenn sie dies einmal thun, so haben sie als solche für den Resorptionsdruck des Zelleninhaltes keine besondere Bedeutung mehr. Durch chemische Umsetzungen in der Zelle werden sie zu complicirteren Eiweissverbindungen aufgebaut, für deren relativ grössere Moleküle die eben durchdrungene Zellenwand nicht weiter durchlässig ist. Folglich müssen sie sodann gleich nach ihrer Entstehung auf die Nährflüssigkeit in dem Sinne osmotisch wirksam sein, dass sie durch die betreffende Wand diese nach sich ziehen und sich schliesslich sammt nachgezogener Flüssigkeit irgendwie in das Drüsenlumen ausgiessen. Aber wie und durch welchen Vorgang?

Betrachten wir die chemischen Analysen des Speichels bei sympathischer und cerebraler Absonderung, so sind einige Verschieden- 
heiten auffallend. Der cerebrale Speichel der Parotis des Kaninchens z. B. enthält 1-2\% Fixa, während der Sympaticus-Speichel etwa $3,7-8,3 \%$ aufzuweisen hat. Diese Differenz wird ausschliesslich durch die Eiweissstoffe bedingt, während der procentige Gehalt an anorganischen Salzen bei den beiden Speichelarten derselhe ist. Dies deutet auf einen verschiedenen Einfluss der resp. Nervenreizung auf die Absonderung einerseits der Eiweissstoffe und andererseits der Salze und des Wassers. Bei Reizung einer frischen Drüse wächst der Gehalt des ausgeschiedenen Speichels an organischen Bestandtheilen rascher als der Wasser- und Salzgehalt, während an der ermüdeten Drüse der umgekehrte Fall eintritt.

Alles dies könnte sich wohl mit der Auffassung im besten Einklang befinden, dass die durch eine besondere Zellenthätigkeit entstehenden specifischen Absonderungsproducte einen osmotischen Flüssigkeitsstrom von der Nährflüssigkeit in das Drüsenlumen mit sich bringen, was allerdings voraussetzt, dass die gegen das Lumen gewandte Zellenwand für die resp. Stoffe durchlässig ist. Nun zeigt aber der Vergleich der anorganischen Salze der Drüsensecrete mit denen der Lympha, dass ihr Gehalt in jener meistens kleiner ist als in dieser. Hier könnte man sich die Sache nach zwei verschiedenen Richtungen hin denken. Die eine Alternative wäre, dass nicht alle in der Nährflüssigkeit gelösten Salze von dieser in die Drüse eindringen können, - eine Vermuthung, die dennoch sehr fraglich erscheint. Denn erstens ist kaum anzunehmen, dass die Salzmoleküle resp. ihre Ionen nicht eine Wand durchdringen könnten, welche für die viel grösseren Moleküle der Eiweisskörper bezw. deren Zerfallsproducte in der Nährflüssigkeit durchlässig wäre. Zweitens repräsentirt der Salzgehalt der Lymphe einen osmotischen Druck von etwa 6,6 Atmosphären oder $5,000 \mathrm{~mm} \mathrm{Hg}$ (von Tammann für das Pferdeblutplasma unter Voraussetzung vollständiger Dissociation der gelösten Bestandtheile berechnet), was somit im Inhalte der secernirenden Drüsenzelle einen ausserordentlichen osmotischen Druck von Seiten der specifischen Zellproducte voraussetzen müsste, damit sie einen der Erfahrung entsprechenden, im Drüsenausführungsgang gemessenen osmotischen Ueberdruck entfalten könnte. Doch möge gerne zugestanden werden, dass sich diese Erwägungen vorläufig auf sehr unsicherem Boden bewegen. Drittens spricht gegen die Undurchlässigkeit der gegen die Nährflüssigkeit gerichteten Zellenwand für die meisten der Salze derselben auch die chemische Analyse der 
ganzen thätigen Drüse, deren Gehalt an Aschenbestandtheilen etwa $1 \%$ [nach Oidtman $]^{1}$ ) beträgt und folglich den der Lymphe an unorganischen Bestandtheilen sogar etwas übertrifft.

Die andere Alternative wäre, die im Drüsenausführungsgange ausgeschiedene Secretionsflüssigkeit als ein Filtrat aus dem Inhalt der thätigen Drüsenzelle aufzufassen. Dass die Secretion unter besonders hohem Drucke stattfindet, ist durch directe Messungen im Drüsenausführungsgange sichergestellt. Es kann somit die Vermuthung nicht befremden, dass der in der thätigen Drüsenzelle obwaltende osmotische Druck solche Dimensionen annehmen konnte, dass eine Filtration nach dem Drüsenlumen hin stattfinden konnte.

Die anatomischen Verhältnisse der Speicheldrüsen, soweit sie uns bekannt sind, würden einer solchen Vermuthung nicht widersprechen. Nach Ramon y Cajol, Rezius, Langerhans u. A. beginnen die Drüsenausführungsgänge mit kleinen Erweiterungen nahe dem Kern innerhalb der Drüsenzellen selbst.

Durch den ausserordentlich hohen intracellularen Druck würden sodann durch die Wände dieser kleinen Gänge, je nach der Durchlässigkeit dieser, Wasser wie auch verschiedene gelöste Stoffe in das Drüsenlumen hindurchgepresst, wobei auch Mengen der specifischen Absonderungsstoffe - vielleicht durch die kleinen Ausführungsgänge selber - mitgerissen würden.

Nun ist es aber eine Frage, ob man die physikalischen Verhältnisse der Drüsenzelle sich so denken kann, dass die unter dem hohen intracellularen Drucke stattfindende Filtration nur nach dem Drüsenlumen hin, nicht aber ebenso durch diejenigen Theile zurück, durch welehe die Nährstoffe bezw. das Wasser aufgenommen worden sind, vor sich gehen sollte. Dies muss sicherlich für möglich gehalten werden, obgleich der nähere Bau der betreffenden Zellenwände wie auch der Mechanismus des Vorganges sich unserem Wissen entziehen. Ganz vernachlässigt darf auch nicht der Umstand werden, dass bei unbehindertem Abfluss des Secretes im Ausführungsgange bezw. im Drüsenlumen ein niedrigerer Manometerdruck herrseht als in der Gewebeflüssigkeit (10-20 mm Sodalösung).

Nach der obigen theoretischen Betrachtung würde die secernirende Thätigkeit der Schleimdrüsen in drei Phasen zergliedert sein. Primär: ein unter versehiedenen physiologischen Einflüssen stehendes Auf-

1) Hammarsten, Lärobok i fysiologisk Kemi p. 182.1883. 
bauen von specifischen Secretbestandtheilen, für welche die an das Nährmaterial angrenzende Zellenwand nicht durchlässig ist; secundär: ein von den specifischen Secretbestandtheilen auf osmotischem Wege aus der Lymphe erfolgendes Aufnehmen von Flüssigkeit, welche einen ausserordentlich hohen intracellularen Manometerdruck zu Wege bringt; und tertiär; ein durch diesen hohen Druck aus der Drüsenzelle in die kleinsten Drüsenausführungsgänge stattfindendes Auspressen oder Filtriren von Flüssigkeit, wobei auch specifische Zellproducte mitgerissen werden.

Ein Blick auf die an der frischen Drüse beobachteten morphologischen Veränderungen zeigt, dass sowohl die Schleimzellen (Biedermann) wie die Eiweisszellen (Langley) in der Rube zum grössten Theile reichlich mit dunklen, lichtbrechenden kleinen Körnchen durchsetzt sird. Bei stark angeregter Secretion - gleichviel, ob dies durch Pilocarpin oder Nervenreizung oder auf physiologischem Wege erzielt wird - fängt der Zelleninhalt von der Acinuswand an, sich aufzuhellen, so dass die Körnchen schliesslich nur an der lumenständigen Wand zu entdecken sind. Während der Thätigkeit sind die Zellen prall gefüllt, nehmen aber besonders nach einer reichlichen Secretion an Grösse ab.

Von dem oben auseinandergesetzten Gesichtspunkte aus würden diese morphologischen Veränderungen folgende Deutung zulassen. Die Drüsenzellen lassen unter gewissen Bedingungen in ihrem Inhalte specifisehe Stoffe entstehen, welche ein Bestreben haben, in Lösung zu gehen. Zu diesem Behufe nehmen sie auf osmotischem Wege durch die Acinuswand von der Lymphe Flüssigkeit auf, wodurch die Zellen stärker gefüllt werden; je nachdem die Lösungsflüssigkeit weiter eindringt, gehen die specifischen Stoffe in Lösung, wobei der Zelleninhalt sich aufhellt und ein gleichmässigeres Aussehen bekommt. Nach stattgefundener Secretion nimmt der Zellenleib an Grösse ah, und sein Inhalt erhält sein protoplasmatisches Aussehen wieder. Gianuzzi's halbmondförmige Protoplasmazellen würden also nach dieser Auffassung in Ruhe verharrende Schleimzellen darstellen.

\section{Schlussbetrachtungen.}

Mit den obigen drei Beispielen will ich gezeigt haben, welche Bedeutung den osmotischen Erscheinungen bei den physiologischen Resorptions- und Secretionsvorgängen theoretisch zukommen muss, 
wenn zwei Lösungen verschiedener Stoffe durch eine Scheidewand getrennt sind, welche für den einen Stoff undurchlässig ist, während der andere Stoff wie auch das Lösungsmittel die Wand mehr oder weniger unbehindert durchdringen können.

Aus diesen Auseinandersetzungen ergibt sich ferner, dass es für das Resorptionsvermögen einer gewissen Lösung in Bezug auf eine andere - keine nothwendige Bedingung ist, dass jene einen Stoff entbält, für welchen die betreffende Scheidewand absolut undurchlässig ist; es genügt, dass die Bewegungshindernisse, welche die Wand dem Diffundiren dieses Stoffes entgegensetzt, sehr gross im Vergleich mit denjenigen sind, denen der andere gelöste Stoff unterliegt. Selbstverständlich wird der Resorptionsdruck, je nach der mehr oder weniger vollständigen Halbdurchlässigkeit einer Scheidewand versehiedene Werthe annehmen und im Laufe des Resorptionsvorganges grosse Veränderungen erfahren, bis früher oder später ein ungefährer Gleichgewichtszustand eintritt und die Resorption in der eingeschlagenen Richtung aufhört.

Eine ganz besondere Bedeutung für die osmotischen Vorgänge insofern sie bei den Resorptions- und Secretionserscheinungen eine Rolle spielen - möchte ich den schwer diffusiblen Eiweissstoffen bezw. den complicirten specifischen Producten der resp. Zellen beigelegt wissen.

Bei dieser Gelegenheit mag auch auf die eventuelle Bedeutung des gelösten Bluteiweisses für das Eindicken des eiweissfreien Glomerulusfiltrates in den Tubuli contorti bei der Nierenthätigkeit aufmerksam gemacht werden.

Die oben entworfene Art der Thätigkeit der zelligen Elemente der Schleimdrüsen dürfte wohl auch cecernirende Drüsenzellen im Allgemeinen charakterisiren. Es findet statt: 1. ein Aufnehmen von Nährmaterial durch die an dasselbe angrenzende Wand und 2. ein Abgeben von specifischen Secretbestandtheilen nebst angehäufter Flüssigkeit durch einen anderen Theil ihrer Hülle bezw. durch die gegen das Drüsenlumen gerichtete Wand. Ob hierbei intermediär ein sehr erhöhter intracellularer Druck im Allgemeinen vorkommt oder nicht, ist nicht von principieller Bedeutung. Wenn aber eine Zelle specifische Secretbestandtheile producirt, die in der umgebenden Gewebeflüssigkeit nicht oder in geringerem Grade vorkommen, so können wir nicht umhin, den osmotischen Erscheinungen einen Spielraum zu gewähren, und da die Secretionsproducte lediglich nach 
einer bestimmten Richtung abgeschieden werden, so müssen ja die physikalischen Bedingungen, welche das Eindringen der Secrete nach ungehörigen Richtungen hin verhindern, vorhanden sein, d. h. die an das Nährmaterial grenzende Zellenwand muss für die specifischen Secretbestandtheile mehr oder weniger undurchlässig, die lumenständige Wand dagegen für sie durchlässig sein.

In der That, welcher Ansicht man auch über den Secretionsmechanismus der Drüsen sein mag, ohne diese beiden Annahmen werden wir nie zurecht kommen können. Von dem mehr oder wenigẹr bevorzugten Abfliessen der Zellensecrete nach dem Drüsenlumen hin dürfte sodann der Betrag des eventuell zum Vorschein kommenden intracellularen Manometerdruckes abhängen, welcher unter Umständen auch so hohe Werthe annehmen kann, dass ein Auspressen, ein Filtriren von einigen Bestandtheilen des Zelleninhaltes stattfinden kann.

Die hier ausgesprochenen Gedanken können in mehrerer Hinsicht einer experimentellen Prüfung zugänglich sein.

Bei einigen Versuchen, die J a c qu e s L o eb ${ }^{1}$ ) zu anderen Zwecken angestellt hat, finden wir Erscheinungen, welche mit den oben in den Drüsenzellen präsumirten analog und für unseren Gegenstand recht lebrreich sind. Loeb liess den Musculus sartorius des Frosches in verschieden concentrirten Lösungen verschiedener Stoffe liegen und mass sodann die Gewichtsveränderungen, welchen der Muskel dabei unterlag. Es stellte sich heraus, dass der Muskel nach kürzerem Liegen in einer gegenüber dem Froschblute hypertonischen Lösung an Gewicht abnahm bezw. in einer hypotonischen Lösung zunahm. Graduelle Verschiedenheiten machten sich bei Lösungen verschiedener Stoffe geltend.

Die Concentration einer NaCl-Lösung, welche in einer Stunde weder $\mathrm{Zu}$ - noch Abrahme des Gewichtes veranlasste, fand er zwischen 0,62 und $0,72 \%$ schwankend. Loeb zieht hieraus den Schluss, dass die osmotischen Eigenschaften thierischer Gewebe und Säfte Schwankungen unterliegen, und hebt hervor, dass in Bezug auf die Isotonie bezw. den osmotischen Druck eine „physiologische Concentration" sich kaum fixiren lässt.

Entschieden bedeutungsvoller für die Auffassung der Erscheinungen, welche sich unter den genannten Bedingungen im Sartorius

1) Jacques Loeb, Physiolng. Untersuchungen über Ionenwirkungen. Pflüger's Archiv Bd. 70. 
abspielen, ist das Ergebniss, dass der Muskel beim Liegen in einer hypertonischen Lösung nach der anfänglichen Gewichtsabnahme später wieder an Gewicht zuzunehmen beginnt.

Dies anscheinend merkwürdige Ereigniss tritt ganz regelmässig bei den Lösungen verschiedenster Salze ein, was von Loeb sachgemäss auf einen im Muskelgewebe allmälig ansteigenden osmotischen Druck zurückgeführt wird.

Woher rührt nun aber dieser allmälig sich einstellende osmotische Ueberdruck im Muskelgewebe, und wie kommt er zu Stande?

Wir könnten uns hier zunächst denken, dass verschiedene Stoffe der Muskelbestandtheile unter der die Muskelsubstanz schädigenden Einwirkung der resp. Lösungen Zerspaltungen erfahren würden, wodurch die molekulare Concentration resp. der osmotische Druck erhöht würde. Wenn aber der erhöhte Druck nur diesem Umstande zuzuschreiben wäre, müsste die Druckerhöhung doch stets nur eine begrenzte sein und kaum Werthe annehmen können, welche den osmotischen Druck der von $\mathrm{Loeb}$ angewandten hyperisotonischen Lösungen übertreffen würden.

Vielmehr müssen wir unser Augenmerk auf die membranartigen Gebilde des Muskels richten, welche das Muskelgewebe von unmittelbarer Berührung mit den resp. Versuchsflüssigkeiten trennen. Fassen wir die graduellen Verschiedenheiten der anfänglichen, das Gewicht herabsetzenden Wirkung hyperisotonischer Lösungen verschiedener Stoffe in's Auge, so können wir den Schluss ziehen, dass alle zum Gebrauch gekommenen Stoffe bezw. ibre Ionen einen anhaltenden osmotischen Wasserstrom aus dem Muskel in die Lösung bewirken. Da nun diese osmotische Wirkung damit zusammenhängt, dass die resp. gelösten Stoffe die Scheidewand nicht oder nur schwer zu durchdringen vermögen, erhellt hieraus, dass die Hüllen des Muskelgewebes für verschiedene Stoffe in verschiedenem Grade durchlässig sind. Je schwerer ein Stoff die Hüllen zu durchwandern vermag, desto intensiver wird er einen osmotischen Strom aus dem Muskel in die Lösung hervorrufen. Dass diese Wirkung den angewandten Stoffen früher oder später wieder abbanden kommt und in eine entgegengesetzte übergeht, beweist somit, dass dieselben schliesslich dennoch alle in den Muskel einzudringen vermögen. Inwieweit während des Versuches pathologische Veränderungen der resp. Hüllen ${ }^{1}$ )

1) Bei Versucherf, die ich unlängst (Pflüger's Archiv Bd. 84 S. 191) bezüglich des elektromotorischen Verhaltens des Froschsartorius angestellt babe, 
mitspielen, ist für unseren jetzigen Gegenstand von untergeordnetem Interesse.

Wo liegt aber nun der Grund des anscheinend unbegrenzt gesteigerten intramuskulären osmotischen Druckes?

Unter den gelösten Bestandtheilen des Muskelgewebes finden sich u. A. auch Eiweissverbindungen, welche bekanntlich besonders wenig diffusible Substanzen darstellen und daher die Muskelhüllen sehr schwer zu durchdringen vermögen. Wird ein Muskel in eine gegenüber der resp. Nährflüssigkeit hypertonische Lösung gebracht, so wird das Wasser des Muskels und der Lösung bestrebt sein, sich dem respectiven in diesen beiden herrschenden osmotischen Drucke proportional zu vertheilen, $d$. $h$. aus den weniger concentrirten Muskelsäften in die Lösung auszuwandern. Je nachdem aber der gelöste Stoff der angewandten Flüssigkeit Zeit bekommt, durch die Muskelhüllen einzudringen, gleicht sich die auf ihn ankommende Concentrationsdifferenz ausser- und innerhalb der Muskelbüllen aus. Dasselbe geschieht in Bezug auf diejenigen löslichen Muskelsubstanzen, welche durch die Muskelhüllen eventuell auszutreten vermögen. Es wird somit nach einer gewissen Zeitfrist ein ungefährer Gleichgewichtszustand hergestellt zwischen allen denjenigen Stoffen, welche sich zu beiden Seiten der Hüllen in Lösung befinden und diese durchdringen können.

Diese Entwickelungsstufe bedeutet aber einen Wendepunkt in den osmotischen Vorgängen des Muskels. Die molekulare Concentration ist nun auf beiden Seiten der trennenden Wand ungefähr gleich gross, aber dennoch nicht ganz gleich. Auf der Innenseite der Scheidewand sind die schwer diffusiblen Eiweissstoffe (eventuell auch andere Substanzen) geblieben und stellen immerwährend einen osmotischen Ueberdruck dar, welcher nicht versäumt, seine Folgeerscheinungen mit sich zu bringen.

Die Ergebnisse der Loeb'schen Versuche, dass der Sartoriusmuskel in einer sowohl hyper- wie hypotonischen Lösung verschiedener Salze früher oder später an Gewicht zunimmt, lassen sich

hat sich als sehr wahrscheinlich herausgestellt, dass die Zerfallsstoffe der specifischen Muskelelemente die völlig ungeschädigten membranartigen Gebilde bezw. Hüllen des Muskelgewebes nicht zu durchdringen vermögen, dass aber mit eintretender Schädigung dieser zunächst die elektropositiven und bei weiter fortgeschrittener Schädigung schliesslich auch die elektronegativen Ionen der Zerfallsstoffe die resp. Hüllen passiren können (S. 236-248). 
also darauf zurückführen, dass im Muskel gelöste Stoffe sich befinden, für welche die Muskelhüllen mehr oder weniger undurchlässig, während sie für das Wasser wie auch für den gelösten Stoff der angewandten Flüssigkeit durchlässig sind.

Wir haben hier also eine Art von Resorptionsvorgang, welcher der Resorption von Salzlösungen aus den serösen Höhlen analog erscheint.

Aus dem beschriebenen Verhalten des Muskels gegenüber verschieden concentrirten Lösungen verschiedener Salze erfahren wir hierbei, dass es keine Concentration gibt, die in der Länge sich gegenüber dem Muskel als isotonisch darstellen würde. Ich habe

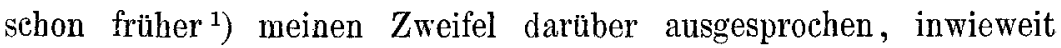
man überhaupt berechtigt sein könnte, von einer mit irgend welchem Gewebe isotonischen Concentration einer Lösung zu sprechen. Der Begriff der Isotonie entspricht streng genommen in seiner ursprünglichen Bedeutung nicht den thatsächlichen Verhältnissen und darf daher nur als Synonym mit "gleiche molekulare Concentration" in Bezug auf Lösungen als solche Verwendung finden. Wo wir es aber mit organisirten Elementen, mit Geweben zu thun haben, deren Grenzschichten bezw. Hüllen für verschiedene Stoffe sowohl der respectiven Lösung wie für die in ihren eigenen Gewebesäften gelösten in verschiedenem Grade durchlässig sind, muss der Begriff der Isotonie immer nur ein sehr relativer sein.

Es dürfte sich wohl schwierig gestalten, auch für einen bestimmten Fall eine gemischte Lösung von geeigneten Componenten mit geeigneten respectiven Partial-Concentrationen ausfindig zu machen, in welcher das betreffende Gewebe seinen Tonus beliebige Zeit unverändert erhalten würde. Für eine solche Lösung würde zweckentsprechend die Benennung "homotonisch" vorzubehalten sein. Als erste Bedingung für die Homotomie einer Lösung in Bezug auf ein gewisses Gewebeelement würde somit gelten, dass die membranartigen Gebilde desselben für die gelösten Stoffe einerseits des Gewebes selber und andererseits der betreffenden Lösung in gleichem Grade durehlässig sind, und dass überdies die die Hüllen verschieden leicht durchdringender Stoffe in geeigneter PartialConcentration vorhanden sind. Die Herstellung einer mit einem Gewebeelemente homotonischen Lösung aus nur einem einzigen ge-

1) Mittheilung I. Pflüger's Archiv Bd. 79 S. 123.1900. 
lösten Stoff und dem Lösungsmittel muss als eine Unmöglichkeit gelten.

Da ich mit dieser Mittheilung bezweckt habe, die Resorptionsund Secretionsvorgänge im Allgemeinen zu besprechen, ist noch Folgendes zu beobachten.

Unter den gelösten Bestandtheilen der thierischen Säfte und Gewebe finden sich überall Salze, d. h. Elektrolyte. Bei ihrer Diffusion erzeugen diese elektromotorische Kräfte $^{x}$ ) bezw. elektrostatische Spannungen, welche davon herrühren, dass die mit ihren respectiven Ladungen von + bezw. -- Elektricität begabten Ionen dank ihrer verschieden grossen Wanderungsgeschwindigkeiten - bei der Diffusion von einander räumlich getrennt werden. Soll ein Elektrolyt durch eine Scheidewand dringen, welche z. B. für das elektropositive Ion des betreffenden Stoffes durchlässig ist, das elektronegative aber nachzufolgen verhindert, so können die hieraus resultirenden elektrostatischen Spannungen relativ gross werden. Werden diese nun durch Nebenschliessungen - wie dies meistens der Fall sein dürfte - ausgeglichen, so ist die Folge, dass ein kataphorischer Flüssigkeitsstrom ${ }^{2}$ ), welcher mit dem positiven Strome gleich gerichtet ist, in der Diffusionsrichtung durch die Scheidewand getrieben wird. Wäre die Scheidewand wiederum für das elektronegative, nicht aber für das elektropositive Ion durchlässig, dann müsste die Diffusionserscheinung unter entsprechenden Bedingungen einen ihr selber entgegengesetzt gerichteten Flüssigkeitsstrom durch die Wand zu Wege bringen.

Die bezüglich der überlebenden Froschhaut gemachte Beobachtung, dass sie, auf beiden Seiten von gleich starker $\mathrm{NaCl}$ Lösung umgeben, die Flüssigkeit von ihrer äusseren Seite nach der inneren treibt, würde in Bezug auf die Richtung mit den theoretischen Betrachtungen übereinstimmen, wenn wir bedenken, dass die Froschhaut der Sitz eines einsteigenden Stromes ist. Hiermit will ich keineswegs gesagt haben, dass nicht andere Factoren von Seiten der

1) Die einschlägigen Lehrbücher der Elektrochemie, sowie meine IV. Mittheilung in diesem Archiv Bd. 84 S. 191 sind zu vergleichen.

2) Näheres hierüber in meinen Veröffentlichungen: Beitrag zur Feststellung einer physik.-chem. Grundlage der elektromedicamentösen Behandlung, 1896, und Experimentelle Untersuchungen über das unter Finwirkung des constanten elektrischen Stromes stattfindende Eindringen von medicamentösen Stoffen in den Thierkörper, 1898. Commissionsverlag von K. F. Koehler, Leipzig. 
Thierische Säfte und Gewebe in physikalisch-chemischer Beziehung. 575

betreffenden Zellen bezw. deren Inbalte eine resorbirende resp. secernirende Thätigkeit entfalten würden.

Welche Bedeutung der Kataphorese bei den Resorptions- und Secretionsvorgängen im Organismus thatsächlich zukommt, lässt sich vorläufig noch gar nicht absehen.

Im Vergleich mit den bedeutenden treibenden Kräften des osmotischen Druckes und in Anbetracht der relativ grossen, für die Kataphorese nachtheiligen Leitfähigkeit thierischer Säfte dürften die kataphorischen Erscheinungen bei den in Rede stehenden Vorgängen von untergeordneter Bedeutung sein. 